In der Rubrik "Literatur kompakt" werden die wichtigsten Originalarbeiten aus der internationalen Fachliteratur referiert.

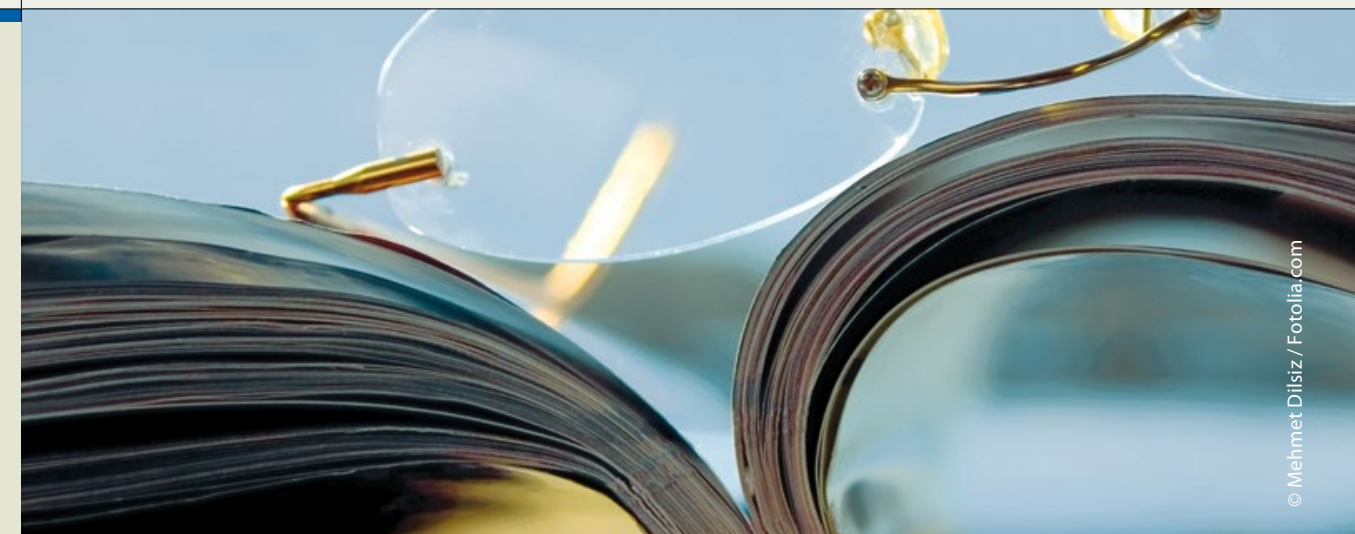

\section{Retinoidtherapie bei Akne: weniger Laborkontrollen nötig}

\section{Aknepatienten unter oraler Isotretinoin-Therapie in Standarddosierung können sich künftig möglicherweise einige Blutentnahmen sparen. Eine Metaanalyse hat jetzt gezeigt, dass das Risiko einer Entgleisung von Leber- und Lipidwerten in der Regel gering ist.}

$B_{s}^{\mathrm{e}}$ ei einer Aknetherapie mit systemischen Retinoiden besteht die Gefahr schwerer Nebenwirkungen. Deshalb sollen während der Behandlung eine Schwangerschaft ausgeschlossen sein und bestimmte Laborwerte kontrolliert werden. Doch in welchen Zeitabständen die Kontrollen nötig sind, um die Sicherheit der Patienten zu gewährleisten, darüber ist man sich bislang noch weitgehend uneinig. Im Rahmen einer Metaanalyse haben Dermatologen aus den USA jetzt den Einfluss der Therapie nommen. auf Lipid- und Leberwerte sowie das Blutbild untersucht. Hierzu wurden 26 Studien mit 1.574 Patienten aufge-

Der mittlere Triglyzeridwert lag während der Therapie bei $119,98 \mathrm{mg} / \mathrm{dl}$, das Gesamtcholesterin bei $184,74 \mathrm{mg} / \mathrm{dl}$, das LDL-Cholesterin bei 109,23 mg/dl und das HDL-Cholesterin bei $42,80 \mathrm{mg} / \mathrm{dl}$. Den Zustand der Leber spiegelten folgende Werte wider: Aspartataminotransferase (AST bzw. GOT): 22,67 U/l, Alaninaminotransferase (ALT bzw.

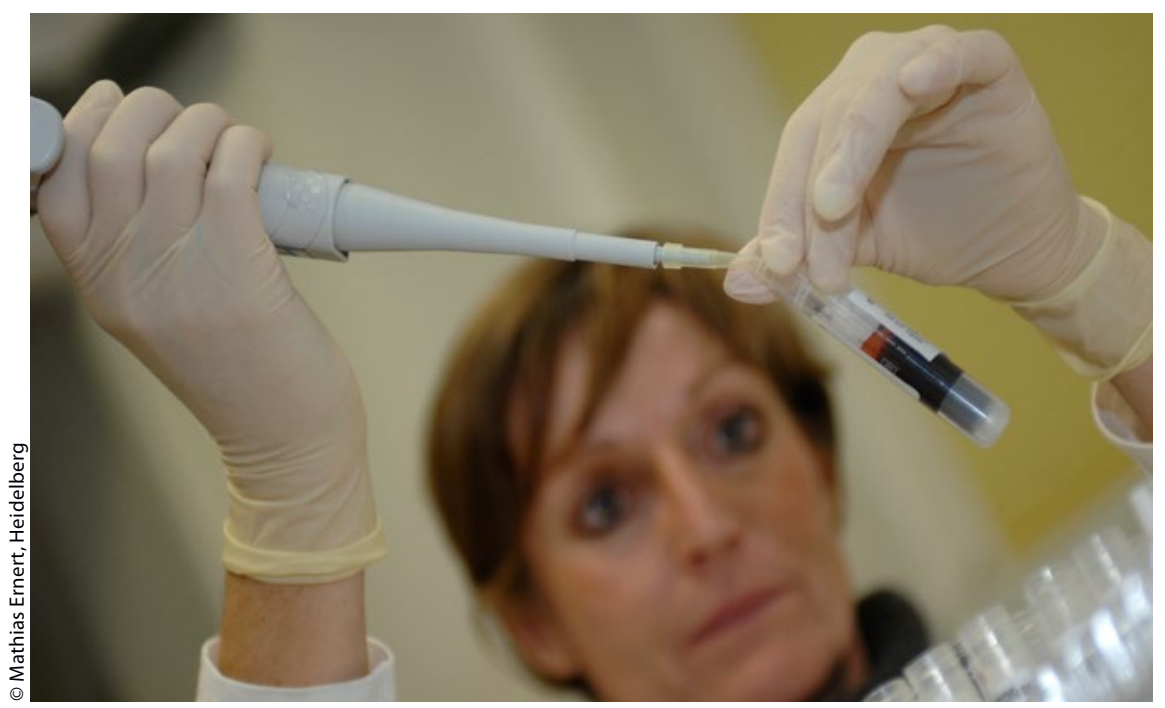

Laborkontrollen können im Rahmen der Retinoidtherapie bei Akne sparsam veranlasst werden.
GPT): 21,77 U/l, Alkalische Phosphatase (AP): 88,35 U/l. Die Leukozytenzahl erreichte durchschnittlich $6.890 / \mu \mathrm{l}$. Im Vergleich zum Ausgangswert waren die Triglyzeride nach acht beziehungsweise 20 Wochen um rund $35 \mathrm{mg} / \mathrm{dl}$ angestiegen. Auch beim Gesamtcholesterin hatten sich leichte Veränderungen ergeben (mittlere Anstiege zwischen 17,72 mg/dl und 23,51 mg/dl). Das LDL-Cholesterin hatte nach 9,7 Wochen um 16,08 mg/dl zugelegt, das HDL war um 4,82 mg/dl gefallen. Auch bei den Leberwerten ergaben sich nach sechseinhalbwöchiger Therapie im Schnitt keine dramatischen Erhöhungen: AST + 3,72 U/L, ALT + 3,22 U/l, AP + 4,23 U/l. Die Leukozytenzahl war nach elfeinhalb Wochen im

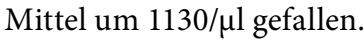

Fazit: In dieser Metaanalyse habe sich zwar eine signifikante Veränderung bei Leukozyten, Leber- und Lipidwerten unter Isotretinoin bestätigt, diese signalisiere aber kein generell hohes Risiko, so die Autoren. Der Anteil der Patienten mit pathologischen Laborwerten sei gering. Deshalb sehen sie bei vielen Aknepatienten mit Standarddosierung keinen Grund für monatliche Kontrollen. Nur wenn ein pathologischer Basiswert oder in der Anamnese Anhaltspunkte für besondere Risiken bestehen, sollten weitere Labortests folgen. Damit spare sich der Patient nicht nur die Aufregung unnötiger Blutentnahmen, dieses Vorgehen wirke auch kostendämpfend, so die Autoren.

Dr. Beate Schumacher

Lee YH et al. Laboratory Monitoring During Isotretinoin Therapy for Acne. A Systematic Review and Meta-analysis. JAMA Dermatol 2016; 152: 35-44 